\title{
Parametrización de una prótesis tumoral de húmero y el desarrollo de un set
}

\author{
Parameterizing a tumor humeral prosthesis and development of a set
}

\author{
Benjamín Araujo-Monsalvo ${ }^{1,2 *}$, Janeth Gómez-Procopio ${ }^{3}$, Víctor M. Araujo-Monsalvo ${ }^{4}$, \\ Luis M. Hernández-Simón ${ }^{1}$, Víctor M. Domínguez-Hernández ${ }^{4,5}$, Genaro Rico-Martínez ${ }^{6}$, \\ Luis M. Linares-González $z^{6}$, Elisa Martínez-Coria ${ }^{7}$ y José L. Nava-Sánchez ${ }^{8}$
}

'Departamento de Ingeniería de Sistemas, Sección de Estudios de Posgrado e Investigación, Escuela Superior de Ingeniería Mecánica y Electtrica, Instituto Politécnico Nacional, Ciudad de México; ${ }^{2}$ Departamento de Ingeniería Biomédica y Mecatrónica, Universidad del Valle de México, Campus Lomas Verdes, Naucalpan, Estado de México; ${ }^{3}$ Dirección de Evaluación Académica, Universidad Tecnológica de México, Ciudad de México; ${ }^{4}$ Laboratorio de Biomecánica, Instituto Nacional de Rehabilitación "Luis Guillermo Ibarra Ibarra", Ciudad de México; ${ }^{5}$ Escuela de Ingeniería y Ciencias, Tecnologico de Monterrey, Campus CCM, Ciudad de M; ${ }^{6}$ Servicio de Tumores Óseos, Instituto Nacional de Rehabilitación "Luis Guiltermo

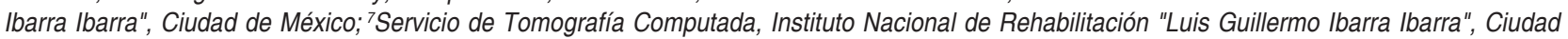
de México; ${ }^{8}$ Tecnológico de Estudios Superiores de Chalco, Chalco, Estado de México. México

\section{Resumen}

Antecedentes: La reconstrucción del húmero proximal es posible a partir de la resección del tumor y la colocación dēuna prótesis. En algunos casos, las prótesis no cumplen con los aspectos antropométricos de los pacientes. Objetivo: Determinar los parámetros para dimensionar los componentes de una prótesis de húmero y el desarrollo de un set, mediante software de diseño. Método: Se seleccionaron 40 pacientes para prótesis, se analizaron radiografías y tomografías computarizadas, y se aplicaron pruebas estadísticas a las mediciones. Resultados: La longitud de los tumores fue de 8-20 cm. La longitud mínima del húmero fue de $28 \mathrm{~cm}$ y la máxima fue de $33 \mathrm{~cm}$. Se observó correlación entre el húmero y el tumor y la altura del paciente, con $p=0.93088$ y $p=0.904564$, respectivamente; para el diámetro del húmero, $p=0.2345$. El set incluye tres componentes, con diámetro de 6-10 mm y longitud de 24, 26 y $28 \mathrm{~cm}$; tres espaciadores modulares con longitudes de 6, 8,5 y $13 \mathrm{~cm}$; coronas de 6,5 y 8,5 cm de largo, 1,3 cm de diámetro y tuerca de $5 \mathrm{~mm}$. Conclusión: Con el desarrollo del set se pueden resolver resecciones mal calculadas y la adaptación a cualquier tamaño de húmero.

Palabras clave: Dimensionamiento. Húmero. Parámetros. Prótesis tumoral. Resección tumoral. Set.

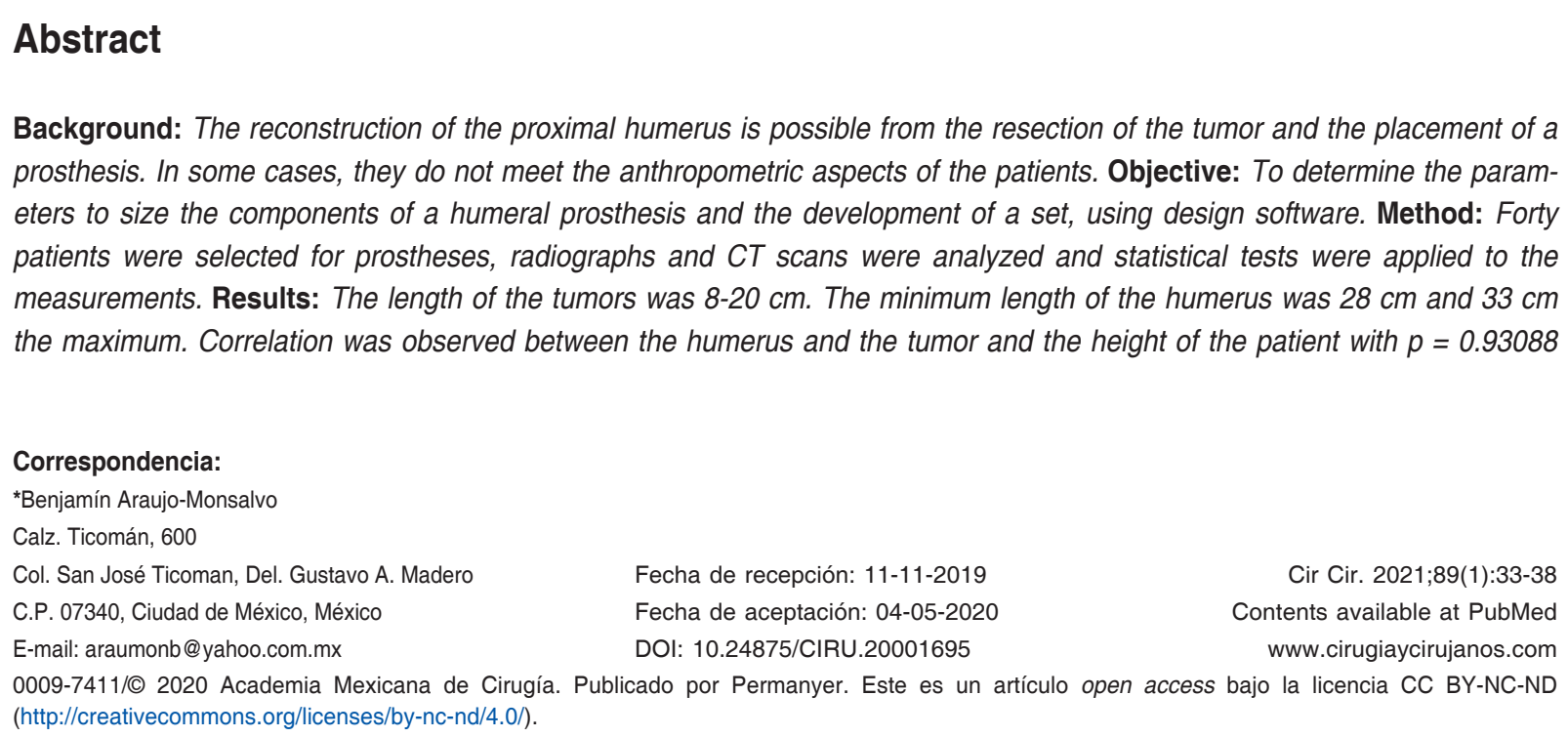


and $p=0.904564$, respectively; humerus diameter, $p=0.2345$. The set will include three components, diameter $6-10 \mathrm{~mm}$, and length of 24, 26 and $28 \mathrm{~cm}$. Three modular spacers with lengths of $6,8.5$ and $13 \mathrm{~cm}$. Crowns 6.5 and $8.5 \mathrm{~cm}$ long, $1.3 \mathrm{~cm}$ in diameter and $5 \mathrm{~mm}$ nut. Conclusion: With the development of the set, poorly calculated resections and adaptation to any humerus size can be resolved.

Key words: Sizing. Humerus. Parameters. Tumor prosthesis. Tumor resection. Set.

\section{Introducción}

El húmero es el tercer sitio más común de presentación de tumores óseos, después de la rodilla y la cadera. En la clasificación de las neoplasias del sistema musculoesquelético, el extremo proximal del húmero ocupa el tercer lugar en afección, sean estas neoplasias benignas, malignas o metastásicas ${ }^{1,2}$.

Debido a que este tipo de lesiones aún conllevan un alto riesgo de complicaciones inherentes al procedimiento quirúrgico, se ha recurrido a diferentes técnicas, como la artrodesis, el uso de endoprótesis, aloinjertos o injertos vascularizados de peroné o de clavícula, aunque ninguno de ellos ha llegado a ser el tratamiento estándar para esta patología $a^{3,4}$.

El aspecto más importante de la cirugía de salvamento de la extremidad por medio de una endoprótesis es preservar las funciones del codo y de la mano, proporcionando una función articular óptima ${ }^{5}$.

Las endoprótesis que existen en el mercado cubren dimensiones de tumores de $3-5 \mathrm{~cm}$, con excelentes resultados al momento de su utilización. Actualmente se han encontrado tumores con dimensiones de 8-20 $\mathrm{cm}$, los cuales no pueden ser cubiertos por las endoprótesis 6 .

Para poder cubrir estas dimensiones de tumores, el Dr. Genaro Rico Martínez diseñó una endoprótesis en el Instituto Nacional de Rehabilitación Luis Guillermo Ibarra Ibarra ${ }^{7-9}$.

Actualmente, el diagnóstico de los pacientes se realiza por medio de radiografía, y su medición por parte del cirujano, seguida de su fabricación por un proveedor. Debido a que solo se tiene un tamaño de endoprótesis, al momento de la cirugía (tratamiento) se observan diferencias anatómicas (endoprótesis vs. paciente), que producen una mala colocación y fijación de la endoprótesis, lo cual lleva a una reprogramación de la cirugía y a la fabricación de una nueva endoprótesis.

La tomografía computarizada (TC) y su reconstrucción tridimensional proveen una imagen anatómicamente más correcta y la visualización tanto externa como interna del húmero, lo que permite la
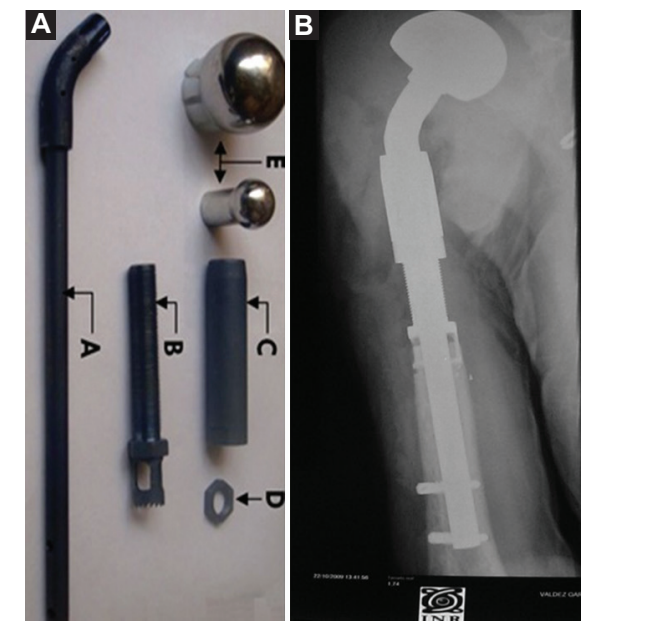

Figura 1. Componentes de la endoprótesis tumoral no convencional de hombro y su colocación. A: vástago. B: corona con cuerda. C: camisa o espaciador modular. D: tuerca. E: cabeza humeral. Diseño del Dr. Genaro Rico Martínez, Instituto Nacional de Rehabilitación MLuis Guillermo Ibarra Ibarra".

estadificación de los tumores, su control evolutivo y aclarar dudas diagnósticas de radiografías y fracturas complejas ${ }^{10}$.

En el Instituto Nacional de Rehabilitación "Luis Guillermo Ibarra Ibarra" se diseñó una endoprótesiș tumoral de húmero RIMAGMR (Osteo Implanhtes Ortopédicos, SA de CV, Puebla, Pue., México)? La endoprótesis está formada por un vástago cilíndrico con un extremo curvo, un espaciador modular expăndible y una corona distal para dar estabilidad y màyor fijación posterior con el crecimiento del hueso a través de ella, a nivel de la osteotomía ${ }^{11}$.

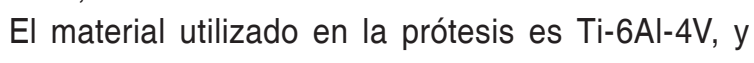
en la cabeza de la prótesis se combina con polietiteno de alta densidad. El peso aproximado de la prótésis humeral oscila entre 350 y $550 \mathrm{~g}$ (Fig. 1).

El sistema de prótesis no convencional RIMAGGM para el húmero proximal cuenta con un cuerpo fijo de 23-26 cm como vástago principal, con orificios en la cara externa de la curva proximal para la inserción musculotendinosa.

La región distal del vástago tiene dos orificios para el bloqueo con pernos de $3.175 \mathrm{~mm}$ (1/8 pulg) de diámetro de separación, un espaciador modular expandible 

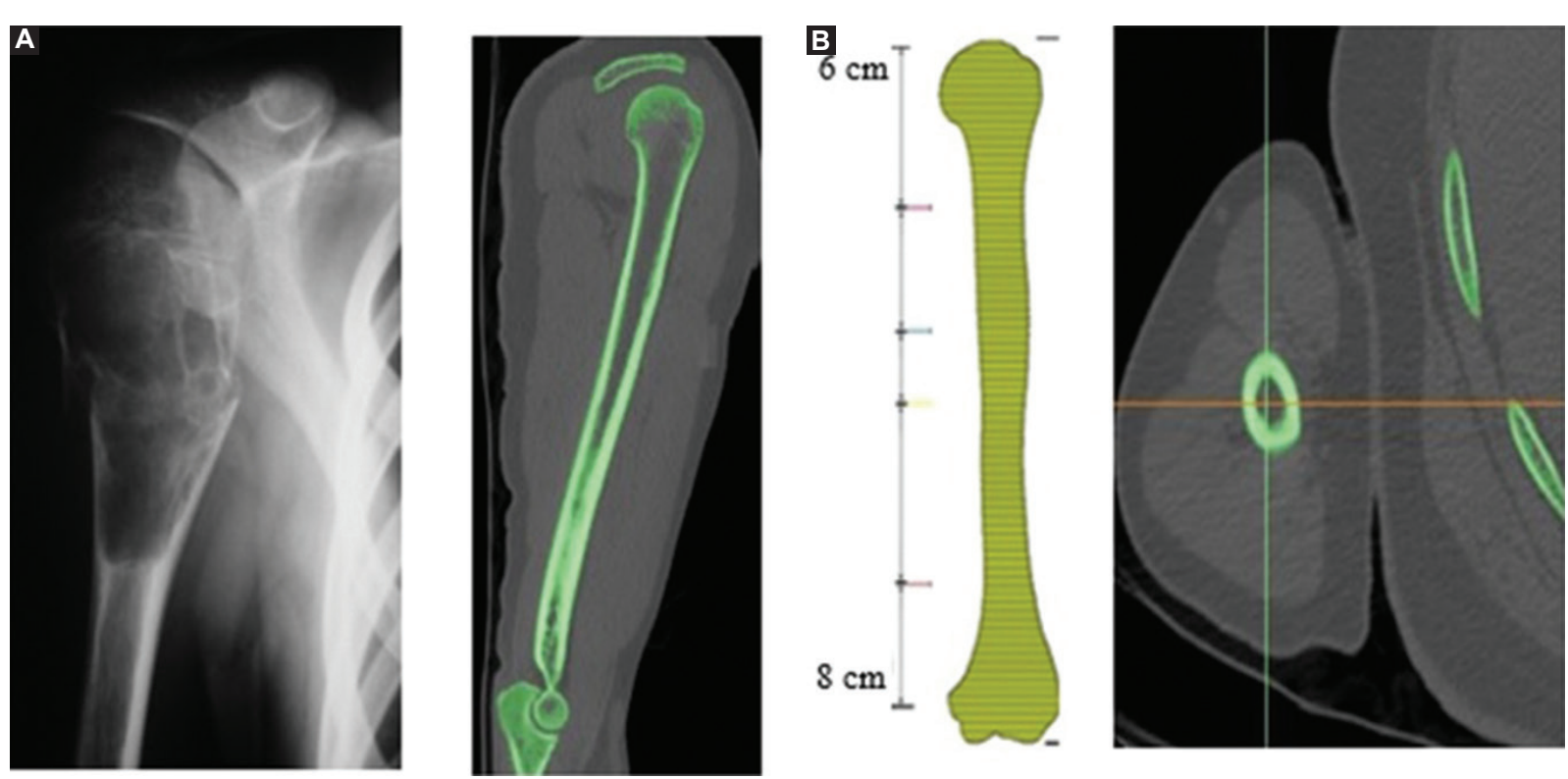

Figura 2. A: radiografía de húmero con tumoración, obtención de dimensiones de tumores existentes en el Instituto Nacional de Rehabilitâción "Luis Guillermo Ibarra Ibarra". B: tomografía computada de húmero sin tumoración de un paciente candidato a prótesis.

mediante tornillo de ajuste (hasta $5 \mathrm{~cm}$ ) con una corona para rodear el hueso en el sitio de la osteotomía y con ventanas para dar más estabilidad entre el binomio hueso-implante, donde posteriormente se espera el crecimiento del hueso a través de la ventana.

El objetivo de este artículo es determinar los parámetros de cada uno de los componentes de la prótesis descrita, estableciendo un estándar en el diseño, y se desarrollará un set que sea capaz de adaptarse a cualquier tamaño de húmero, reconstruir hasta el tercio distal del húmero y permitir resolver resecciones mal calculadas.

\section{Método}

Se realizó un estudio en el que se incluyeron pacientes de cualquier edad y sexo, con expediente completo PACS-INR (Picture and Communication System del Instituto Nacional de Rehabilitación Luis Guillermo Ibarra Ibarra) y consentimiento informado firmado, con tumores benignos agresivos, malignos, tumores de origen medular y metastásicos, tumores benignos agresivos en etapa 3 de Enneking, tumores malignos intracompartimentales (grados IIA y IIB) y metastásicos (grado III). Del estudio, se seleccionaron 40 pacientes candidatos a prótesis.

Los pacientes contaron con radiografías convencionales de húmero en posición anteroposterior y lateral para la medición de la longitud y del diámetro de la tumoración por parte del médico, y con TC para la medición de la longitud del húmero sano, así como el canal medular (Fig. 2 A y B).

Se utilizó la clasificación quirúrgica de Malawer para identificar lesiones intraarticulares de tipo lis Se utilizó el sistema de estadiaje de Enneking y se âplicaron los resultados de la Sociedad de Tumores Musculoesqueléticos para la colocación de la endoprótesis y la reconstrucción de los tejidos blandos posteriör a la resección en bloque del tumor.

Las TC se realizaron con un equipo Multicorte GE Light Speed de 64 cortes (GE Light Speed, General Electric Company, Fairfield, CT, USA), área azulcon estación de trabajo y visualizadores de imagen.

Se obtuvieron imágenes en formato DICOM (Digital Imaging and Communication in Medicine) a partir de un estudio tomográfico y se procesaron con el šsoftware Mimics Innovation Suite (Materialise Inc. Leeuven, Bélgica), versión 17.

Por medio del software se realizó la medición de los húmeros para su clasificación (chico, mediäno, grande) y el conocimiento de las dimensiones (löngitud y diámetro). Además, se realizó la limpieza del canal medular de las TC para una medición más exacta del diámetro de los cortes tomográficos. $\frac{\cup}{\sigma}$

Los parámetros de medición de la TC se obtuvieron a partir de $8 \mathrm{~cm}$ de la zona distal y $6 \mathrm{~cm}$ de la zona proximal, cada 10 cortes a partir de estas. Se dïseñaron polilíneas y se crearon curvas (Fig. $3 \mathrm{~A}$ y B).

Los cortes tomográficos obtenidos se trabajarōn en 3-matic (Materialise Inc., Leuven Bélgica) para la 

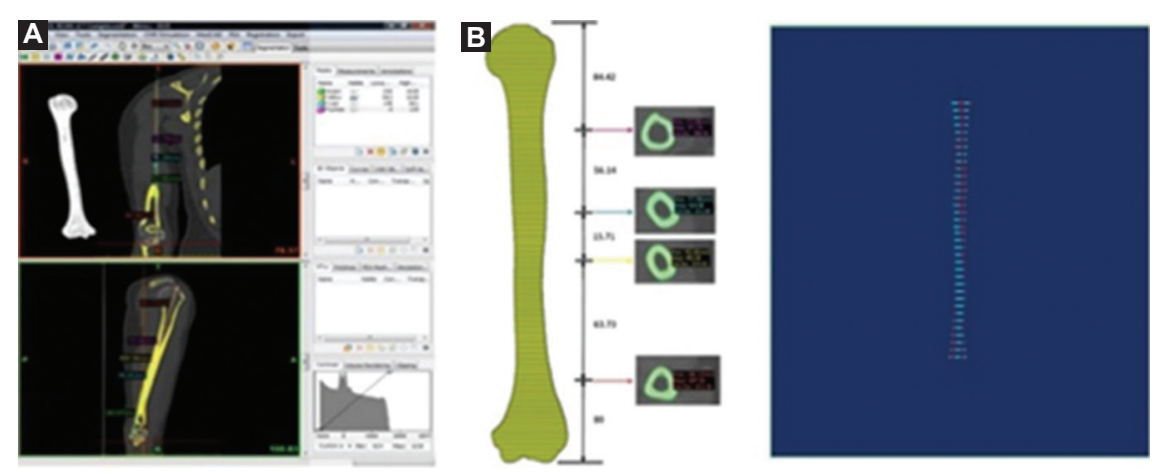

Figura 3. A: cortes tomográficos realizados con el software. B: creación de polilíneas como guía para la limpieza y la medición de las tomōgrafías. Software: Mimics, 3-matic (Materialise Inc., Leuven Bélgica).

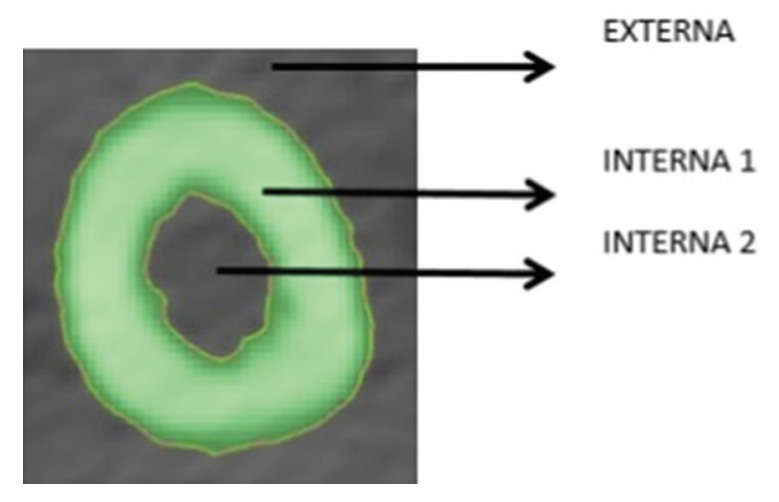

Figura 4. Medición de los cortes tomográficos del húmero (longitud y diámetro), limpieza del canal medular de tomografías computadas para su medición ( $8 \mathrm{~cm}$ distal y $6 \mathrm{~cm}$ proximal). Creación de polilíneas, curvas y medición. Software: Mimics, 3-matic (Materialise Inc., Leuven Bélgica).

medición del diámetro y la recopilación de los datos. De las imágenes obtenidas por el software, se realizaron tres mediciones del canal medular: interna (diámetros de los vástagos), interna (diámetros de la corona) y externa (diámetro del implante), además de la longitud del húmero (Fig. 4).

Se realizaron pruebas estadísticas con el software Statgraphics Centurion (Statpoint Technologies, Inc., Warrenton, Virginia, USA), versión 16.1.11; se aplicó la prueba de Kolmogorov-Smirnov para determinar la distribución de los datos.

La correlación entre las variables se evaluó utilizando la prueba de análisis de regresión lineal y la prueba ANOVA, así como el análisis múltiple de rangos (LSD [Least Significant Difference] 95\%), para la obtención de las dimensiones de cada componente de la prótesis y el número de piezas que componen el set. Todo ello con un nivel de confianza del $95 \%$ y un error del $5 \%$.

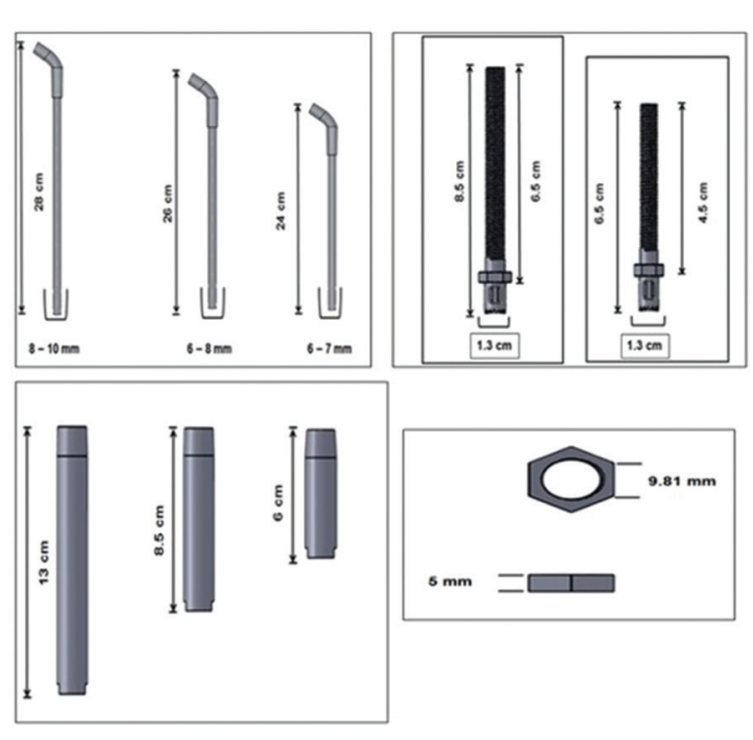

Figura 5. Dimensiones de los componentes de la prótesis, y composición del set.

\section{Resultados}

De los estudios radiológicos se obtuvo la longitud de los tumores $(8-20 \mathrm{~cm})$. De acuerdo con las TE, el húmero de menor longitud era de $28 \mathrm{~cm}$ y el de mâyor longitud era de $33 \mathrm{~cm}$. Del análisis estadístico sęoptuvo la distribución de los datos, así como el cöeficiente de correlación de los datos entre la longitudadel húmero, la estatura del paciente y la longitud defftumor (Tabla 1).

Las dimensiones de cada componente (longitud y diámetro), así como el número de piezas que componen el set, se muestran en la tabla 2.

El set contará con tres vástagos, con un diámètro de 6-10 $\mathrm{mm}$ y una longitud de $24 \mathrm{~cm}$ para húmeros chicos, $26 \mathrm{~cm}$ para húmeros medianos y $28 \mathrm{~cm}$ para 
Tabla 1. Distribución de los datos y coeficiente de correlación de las variables de estudio

\begin{tabular}{|c|c|c|c|c|}
\hline \multirow[t]{2}{*}{ Variable } & \multirow[t]{2}{*}{ Normalidad ${ }^{*}$} & \multicolumn{2}{|c|}{ Variables } & \multirow{2}{*}{$\frac{\text { Resultados }^{\dagger}}{\mathbf{c C}^{\dagger} \bar{c}}$} \\
\hline & & Dependiente & Independiente & \\
\hline EP & 0.9285 & $\mathrm{LH}$ & $\mathrm{EP}$ & 0.93088 \\
\hline LH & 0.9036 & $\mathrm{LH}$ & LT & 0.904564 \\
\hline DV & 0.8472 & $\mathrm{LH}$ & $\mathrm{D}$ & 0.23457 \\
\hline DE & 0.8591 & & & $: \overline{\frac{0}{2}}$ \\
\hline DV hc, hm, hg & $0.8903,0.9015,0.9174$ & & & $\frac{\text { 응 }}{0}$ \\
\hline DE hc, hm, hg & $0.9056,0.9287,0.9483$ & & & 르 \\
\hline
\end{tabular}

CC: coeficiente de correlación; D: diámetro; DE: diámetro externo; DV: diámetro del vástago; EP: estatura del paciente; hc: húmero chico; hm: húmero mediano; hg: húmero grande; $\longleftarrow$ LH: longitud del húmero; LT: longitud del tumor.

*Prueba Kolmogórov-Smirnov.

†Análisis de regresión lineal.

Tabla 2. Dimensiones y número de piezas. Vástago, corona con cuerda, espaciador modular

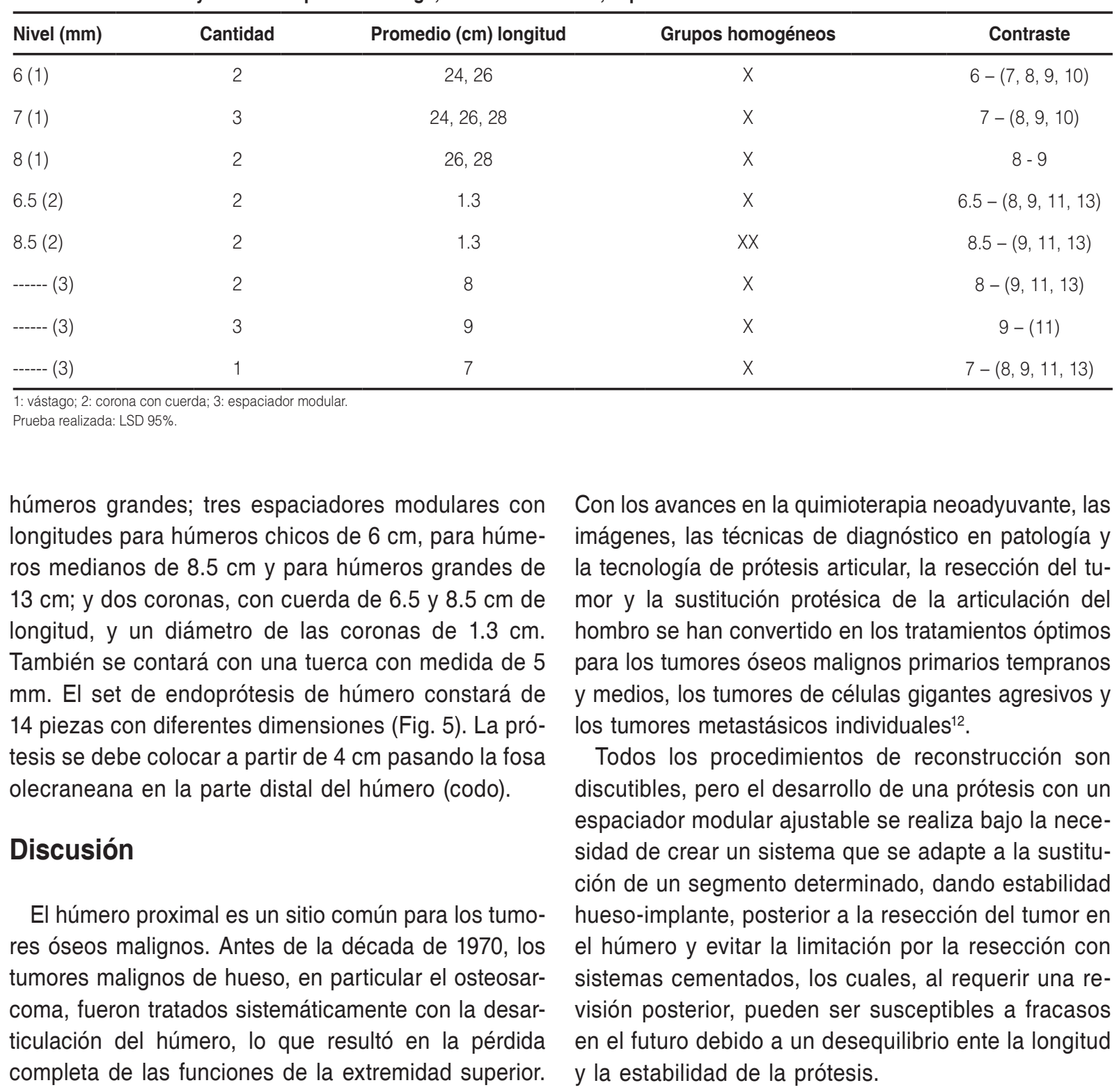


La prótesis de reemplazo de la articulación del hombro (endoprótesis) incluye la prótesis proximal de húmero con diferentes componentes y diversas dimensiones, la cual en su conjunto comprende toda la articulación del hombro. Con relación a la sobrevida de este implante, aproximadamente el $70 \%$ de los pacientes han tenido buen resultado, o dicho de otra forma, el tipo de resección y la reconstrucción de los tejidos blandos es probablemente más importante que el tipo de implante ${ }^{13,14}$.

El sistema de prótesis no convencional RIMAGMR para el húmero proximal cuenta con un cuerpo fijo de $23-26 \mathrm{~cm}$ como vástago principal, con orificios en la cara externa de la curva proximal para la inserción musculotendinosa.

La región distal del vástago tiene dos orificios para el bloqueo con pernos de diámetro $3.175 \mathrm{~mm}$ (1/8 pulgada) y con $25.4 \mathrm{~mm}$ de separación, un espaciador modular expandible mediante tornillo de ajuste hasta de $5 \mathrm{~cm}$, con una corona para rodear el hueso en el sitio de la osteotomía y con ventanas para dar más estabilidad al binomio hueso-implante, donde posteriormente se espera el crecimiento del hueso a través de la ventana. En casos indispensables también se puede utilizar cemento (hasta el momento no se ha utilizado en nuestra serie).

La realización de radiografías aunada a las TC para la parametrización de la prótesis tumoral no convencional de húmero y el dimensionamiento de cada uno de sus componentes para el desarrollo de un set surgen como opción para el tratamiento de tumores en pacientes que ingresan al Instituto Nacional de Rehabilitación Luis Guillermo Ibarra Ibarra15,16.

Las pruebas estadísticas realizadas para el desarrollo del set tienen la finalidad de que el especialista cuente con una gama de prótesis con diferentes dimensiones para la toma de decisiones que impacten positivamente en los pacientes. Esto da la pauta para, en trabajos futuros, realizar un rediseño de la prótesis, la cual comprenda un vástago de forma cónica además del rediseño de la corona con cuerda.

\section{Agradecimientos}

Los autores agradecen el trabajo realizado por el técnico Martín Luna Méndez, del servicio de tomografía computada del Instituto Nacional de Rehabilitación Luis Guillermo Ibarra Ibarra.

\section{Financiamiento}

Los autores declaran que no hubo fuentes de financiamiento.

\section{Conflicto de intereses}

Los autores declaran no tener conflictos de intereses relacionados con el presente manuscrito.

\section{Responsabilidades éticas}

Protección de personas y animales. Los autores declaran que para esta investigación no se han realizado experimentos en seres humanos nianimales.

Confidencialidad de los datos. Los autores deelaran que han seguido los protocolos de su centro de trabajo sobre la publicación de datos de pacientess.

Derecho a la privacidad y consentimiento informado. Los autores han obtenido el consentimiento informado de los pacientes y/o sujetos referidos en el artículo. Este documento obra en poder del autoÉde correspondencia.

\section{Bibliografía}

1. Cortés R, Lezama P, Rubio JA, Domínguez J, Gámiz CA, Pablo Ädrián Carrillo PA. Osteosarcoma. Cirugía de preservación de extremidād torácica con prótesis tumoral no convencional RC-8. Reporte de un Easo. Arch Inv Mat Inf. 2012;4:43-7.

2. Daigeler A, Lehnhardt M, Dhadra A, Hauser J, Steinstraesser L, Langer $\mathrm{S}$, et al. Proximal major limb amputations - a retrospective analysis of 45 oncological cases. World J Surg Oncol. 2009;7:15.

3. El-Sherbiny M. Reconstruction of the proximal humerus after wide resection of tumors: comparison of three reconstructive options. J Egypt Nat Cancer Inst. 2008;20:369-78.

4. Irlenbusch U, Gebhardt K, Rott O, Werner A. [Reconstruction of the rotational centre of the humeral head depending on the prosthetic design.] Z Orthop Unfall. 2008;146:211-7.

5. Kiliç M, End S, Irlenbusch U. Replication of the rotational center of the humeral head with second-generation stemmed prostheses. Acta Opthop Traumatol Turc. 2011;45:71-8.

6. Kiss J, Sztrinkai G, Antal I, Szendro M. Functional results and quality of life after shoulder girdle resection in musculoskeletal tumors. J Shoulder Elbow Surg. 2007;16:273-9.

7. Kitagawa Y, Thai DM, Choong PFM. Reconstructions of the shoulder following tumour resection. J Orthop Surg. 2007;15:201-6.

8. Linares LM, Rico G, Domínguez VM, Araujo V, Delgado EA, León SR. Tratamiento y reconstrucción en tumores óseos del húmero con prótesis no convencional tipo RIMAG. Acta Ortopédica Mexicana. 2014,28:3-11.

9. Masamed R, Learch TJ, Menendez LR. Bloc shoulder resection with total shoulder prosthetic replacement: indications and imaging findings. AJR. 2008;191:482-9.

10. Pearl ML, Kurutz S, Postachini R. Geometric variables in anatomic replacement of the proximal humerus: how much prosthetic geometry is necessary? J Shoulder Elbow Surg. 2009;18:366-70.

11. Rödl RW, Gosheger G, Gebert C, Lindner N, Ozaki T, Winkelmaøn W. Reconstruction of the proximal humerus after wide resection of tumours. J Bone Joint Surg Br. 2002;84:1004-8.

12. Scotti C, Camnasio F, Peretti GM, Fontana F, Fraschini G. Modular prostheses in the treatment of proximal humerus metastases: review of 40 cases. J Orthopaed Traumatol. 2008;9:5-10.

13. Shi SF, Dong Y, Zhang CL, Bao K, Ma XJ. Prosthetic replacement of the proximal humerus after the resection of bone tumors. Chin $\mathrm{J}$ Cancer. 2010;29:121-4.

14. Teunis T, Nota S, Hornicek FJ, Schwab JH, Lozano S. Outcomeafter reconstruction of the proximal humerus for tumor resection: a systematic review. Clin Orthop Relat Res. 2014;472:2245-53.

15. Van de Sande MAJ, Sander DPD, Taminiau AHM. Proximal humerus reconstruction after tumour resection: biological versus endoprosthetic reconstruction. Int Orthop (SICOT). 2011;35:1375-80.

16. Zeballos J. Fibrosarcoma proximal de húmero, salvataje de miembro, reconstrucción con endoprótesis no convencional Fabroni. Revista Médic. 2000;11(17). 